\title{
On gradient-based optimization of jacket structures for offshore wind turbines
}

\author{
Oest, Jacob; Sandal, Kasper; Schafhirt, Sebastian; Stieng, Lars Einar S.; Muskulus, Michael
}

Published in:

Wind Energy

Link to article, DOI:

10.1002/we.2206

Publication date:

2018

Document Version

Peer reviewed version

Link back to DTU Orbit

Citation (APA):

Oest, J., Sandal, K., Schafhirt, S., Stieng, L. E. S., \& Muskulus, M. (2018). On gradient-based optimization of jacket structures for offshore wind turbines. Wind Energy, 21(11), 953-967. https://doi.org/10.1002/we.2206

\section{General rights}

Copyright and moral rights for the publications made accessible in the public portal are retained by the authors and/or other copyright owners and it is a condition of accessing publications that users recognise and abide by the legal requirements associated with these rights.

- Users may download and print one copy of any publication from the public portal for the purpose of private study or research.

- You may not further distribute the material or use it for any profit-making activity or commercial gain

- You may freely distribute the URL identifying the publication in the public portal

If you believe that this document breaches copyright please contact us providing details, and we will remove access to the work immediately and investigate your claim. 


\title{
On gradient-based optimization of jacket structures for offshore wind turbines
}

\author{
Jacob Oest ${ }^{1}$, Kasper Sandal ${ }^{2}$, Sebastian Schafhirt ${ }^{3}$, Lars Einar S. Stieng ${ }^{3}$, Michael Muskulus ${ }^{3}$ \\ ${ }^{1}$ Department of Materials and Production, Aalborg University, Aalborg East, Denmark \\ ${ }^{2}$ Department of Wind Energy, Technical University of Denmark, Risø Campus, Roskilde, Denmark \\ ${ }^{3}$ Department of Civil and Environmental Engineering, Norwegian University of Science and Technology NTNU, Trondheim, Norway
}

\begin{abstract}
During the bidding or very early design phases of jacket structures for offshore wind turbines, there may be very limited information available on meteorological conditions, soil conditions, turbine specifications etc. However, it is still important to quickly produce near-optimal designs with production costs similar to that of the final support structure. Numerical optimization methods can be used to this purpose. This paper investigates three gradient-based optimization methods where preliminary designs are produced by mass optimization. The mass is reduced by changing tube diameter and thickness of the structural members, and the optimization considers both frequency and fatigue constraints. The three methods are based on (i) damage equivalent loads, (ii) quasi-static analysis, and (iii) dynamic analysis. The optimizations are carried out using in-house software JADOP (JAcket Design OPtimization), and the optimized designs are evaluated using state-of-the-art integrated time-domain simulation software FEDEM Windpower. The findings show that each analysis can be applied with success. However, if excitations of structural frequencies contribute significantly to the overall damage, special care must be taken with quasi-static and static modeling. It is observed that wave-loading does not contribute considerably to the fatigue damage. Additionally, the aerodynamic loading does not change significantly with changes of tube geometry within the optimization ranges. The optimized designs are partly driven by reducing stress concentration factors, which can be achieved by reducing the chord diameter to thickness ratio. Thus, the optimized designs resemble each other to a certain extent. Copyright (c) 0000 John Wiley \& Sons, Ltd.
\end{abstract}

\section{KEYWORDS}

offshore wind; jacket support structures; optimization; analytical sensitivities; fatigue

Correspondence

J. Oest, Department of Materials and Production, Aalborg University, Fibigerstraede 16, 9220 Aalborg East, Denmark.

E-mail: oest@mp.aau.dk

Received...

\section{INTRODUCTION}

Wind energy is a reliable and important supplier of sustainable energy. In favorable site conditions, the levelized cost of energy can be comparable to conventional energy sources. However, such site-conditions are limited. Off-coastal areas often present great wind conditions and space for large wind-farms, but offshore wind energy is in general more expensive than onshore wind energy [1,2]. In deep waters, jacket structures are generally considered the most cost-effective bottomfixed support structures. Deep water conditions increase the levelized cost of energy due to larger support structures, more expensive infrastructure, and higher installation and maintenance costs. Support structures account for about $19 \%$ of the total cost of an offshore wind farm [3]. Design, manufacturing, and installation of support structures have been identified as areas of large cost saving potential $[4,5]$.

Human designers are often restricted to a very limited number of design iterations due to time- and cost-related considerations. Computer-aided structural optimization techniques are not restricted in the same manner. For this reason, structural optimization techniques are increasingly being applied in wind energy. In general, there are two types of numerical optimization techniques that can be applied. There are heuristic methods and gradient-based methods. Both methods have their strengths and weaknesses, and both methods have been applied to optimization of support structures. 
Heuristic methods are update schemes often based on analogies from behaviors observed in nature. Heuristic methods rely on function values alone and are therefore also referred to as gradient-free methods. However, as the methods do not utilize design sensitivities, they often require many design iterations before convergence.

Some of the first work in the field of structural optimization of wind turbine support structures was using gradientfree methods to optimize monopile structures [6]. Since then, much work has been done on optimizing both onshore and offshore monopile structures using gradient-free methods [7, 8, 9]. As jackets gained in popularity, different gradient-free methods have also been applied in the design thereof $[10,11,12,13,14]$. A different approach to efficient optimization is shown in [15]. By using a two-stage approach where the optimization of tube geometry and Stress Concentration Factors (SCFs) are assumed independent of the structural analysis, the computational effort needed in the jacket optimization is greatly reduced.

Gradient-based optimization methods often converge in fewer iterations than gradient-free optimization methods. However, at least first order derivatives of cost and constraint functions are necessary. In [16] a method of gradient-based optimization of tower and turbine to reduce the levelized cost of energy is presented. In the optimization, stress, fatigue, and frequency constraints on both tower and turbine are considered. The design sensitivities are estimated using finite difference approximations. The optimization framework was further developed in [17], where both the wind farm layout and the offshore monopile are optimized, again using finite difference approximations of the design sensitivities. Using finite difference schemes can be efficient, but care must be taken as the gradients can be very sensitive to perturbation size $[18,19]$.

Analytical gradient-based optimization of a jacket structure is solved in [20,21], where a dynamic analysis is employed. The optimization reduces the overall mass subject to Ultimate Limit State (ULS) constraints, to Fatigue Limit State (FLS) constraints, and to frequency constraints. In [19] analytical gradient-based optimization of a jacket is solved using a quasistatic analysis to lower the computational costs. For a more detailed overview of optimization of support structures, see [22].

The designer of the support structure may be different from the designer of the tower and turbine. Thus, it may prove time-consuming to obtain updated loading conditions when the substructure is altered. The challenge is to design a jacket based on initial information, that has an accumulated damage near the fatigue limit when re-evaluated with updated loading conditions. This paper investigates this problem using three state-of-the-art analytical gradient-based sizing optimization methods with fatigue and frequency constraints. All three methods utilize linear finite element theory and beam elements. Additionally, all three methods use the Hot Spot Stress (HSS) method where SCFs are applied according to the recommended practice [23]. The three different approaches can be classified by their respective structural analysis method. In other words, the approaches are using (i) a static analysis, (ii) a quasi-static analysis, and (iii) a dynamic analysis. The approach in (i) uses Damage Equivalent Loads (DEL) [24]. It follows that a static analysis is much faster than a quasi-static analysis. In turn, the quasi-static analysis is faster than the dynamic analysis. However, it also follows that the dynamic analysis is more accurate than the quasi-static analysis and the static analysis.

The applied optimization formulations do not include ULS constraints as emphasis in this study is on fatigue modeling and optimization. Introducing ULS constraints will produce much more realistic designs. However, active ULS constraints may hinder tendencies and pitfalls in the fatigue optimization problem which is the main study of this work.

Neither of the three optimization approaches attempt to produce fully validated and readily producible designs, but rather very good preliminary designs. In this framework, a good design is defined as having a low mass and fatigue damage levels near the desired value when reevaluated using state-of-the-art integrated time-domain simulations, here performed with the commercial software FEDEM Windpower (Fedem Technology AS, Trondheim, Norway, Version R7.2.1). It must be noted that the optimized designs should not be directly compared to the original reference design, as the design basis is completely different. The reference design, the so-called OC4 jacket, was designed to compare time simulation codes and does not represent a realistic wind turbine substructure. The presented optimized designs do not represent realistic designs either as many important design criteria such as ULS and manufacturing are excluded in this study. Additionally, a realistic design should be optimized with respect to levelized cost of energy and not just overall mass.

\section{METHODS}

The applied design process can be described using a flow chart, see Figure 1. An initial design is used to generate a finite element representation of the structure in JADOP. This structure is exported to FEDEM Windpower using the application programming interface. In FEDEM Windpower, the jacket is attached to a predefined wind turbine. Next, a transient analysis of the entire structure is performed and the results are extracted. The results are used to deconvolute the loading conditions, such that forces and moments corresponding to the wind-induced loads can be applied in JADOP. Then, the optimization problems are formulated in JADOP, where tube diameters and thicknesses are optimized using IPOPT (Interior Point OPTimizer, [25]). The optimized design is reevaluated in FEDEM Windpower using a full transient 


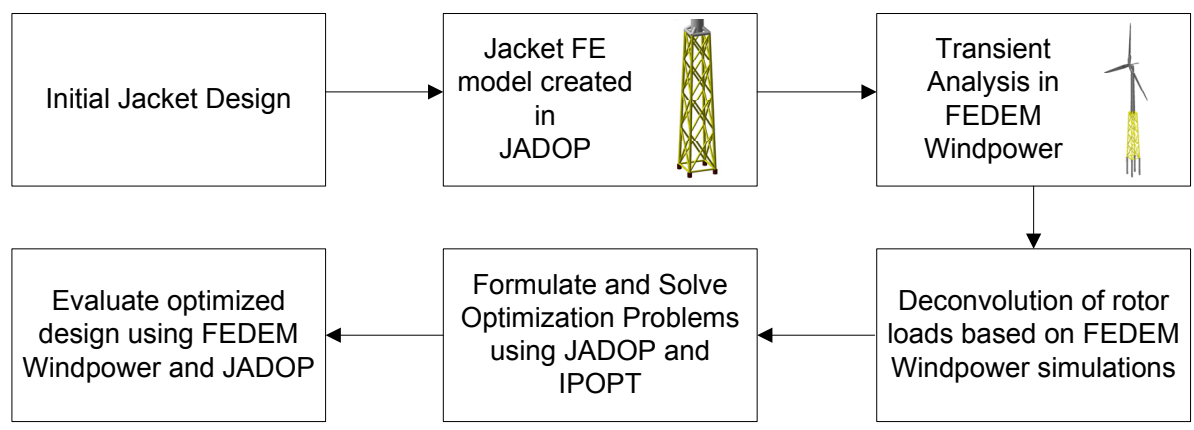

Figure 1. The applied design process.

multibody analysis with an integrated turbine and recalculated wave-loads. Nodal displacements from the integrated analysis are post-processed in JADOP such that SCFs can be used in the fatigue analysis.

To achieve fully optimized designs with respect to fatigue, the optimization process can be restarted using updated rotor and wave loads. At this stage, it is recommended to include more constraints such as ULS and manufacturing constraints. This is not a focus area in the present work. Thus, the presented work can be considered a benchmark of the three methods rather than an attempt to produce a fully optimized design.

\subsection{Structural analysis}

A linear elastic finite element analysis is used, and the jacket is modeled using two-node Timoshenko beam elements with six degrees-of-freedom in each node [26]. Each beam element has a constant cross-sectional area defined by the diameter and thickness of the circular hollow tube. The equilibrium at time $t$ is:

$$
\boldsymbol{M}(\boldsymbol{x}) \ddot{\boldsymbol{U}}(\boldsymbol{x}, t)+\boldsymbol{C}(\boldsymbol{x}) \dot{\boldsymbol{U}}(\boldsymbol{x}, t)+\boldsymbol{K}(\boldsymbol{x}) \boldsymbol{U}(\boldsymbol{x}, t)=\boldsymbol{P}(t)
$$

$\boldsymbol{x}$ is the vector of design variables (diameters and thicknesses), $\boldsymbol{M}$ is the mass matrix, $\boldsymbol{C}$ is the damping matrix, and $\boldsymbol{K}$ is the stiffness matrix. $\boldsymbol{P}$ is the load vector assumed independent of design changes. $\boldsymbol{U}, \dot{\boldsymbol{U}}$, and $\ddot{\boldsymbol{U}}$ are the vectors of global displacements, velocities, and accelerations, respectively. In the quasi-static approach, the mass and damping terms are omitted. A consistent mass matrix $\boldsymbol{M}$ is applied. The $\alpha$ and $\beta$ parameters of the Rayleigh damping matrix $\boldsymbol{C}$ is tuned to $1 \%$ critical damping at the first eigenfrequency of the structure without the Rotor-Nacelle-Assembly (RNA) attached. Timeintegration is performed using Newmark- $\beta$ with constant average acceleration. The soil-structure boundary is simplified as fixed. The analyses in JADOP and FEDEM Windpower have been compared and differences in displacements are generally $<1 \%$.

Jacket structures are dominated by normal stresses. Thus, the recommended practice for fatigue assessment in welded tubular joints combines normal stresses with SCFs [23]. SCFs are design-dependent scaling factors. There are separate SCFs to scale damage caused by normal stress from normal forces $(\mathrm{N})$, from in-plane bending moments (MIP), and from out-of-plane bending moments (MOP). The normal stresses in each element $e$ is:

$$
\left[\begin{array}{c}
\sigma_{e}^{\mathrm{N}}(\boldsymbol{x}, \boldsymbol{U}(\boldsymbol{x}, t)) \\
\sigma_{e}^{\mathrm{MIP}}(\boldsymbol{x}, \boldsymbol{U}(\boldsymbol{x}, t)) \\
\sigma_{e}^{\mathrm{MOP}}(\boldsymbol{x}, \boldsymbol{U}(\boldsymbol{x}, t))
\end{array}\right]=\boldsymbol{E} \boldsymbol{B}_{\boldsymbol{x} \boldsymbol{x}}(\boldsymbol{x}) \boldsymbol{u}_{e}(\boldsymbol{x}, t), \quad \forall e
$$

$\boldsymbol{E}$ is the constitutive matrix, $\boldsymbol{B}_{x x}$ is the strain-displacement matrix including only terms regarding normal stresses, and $\boldsymbol{u}_{e}$ is the element displacement vector. Let $x, y, z$ be local element coordinates, then the sampling locations must be as shown in Figure 2. The scaled stress $\sigma_{i}$ for location $i$ is:

$$
\sigma_{i}(\boldsymbol{x}, \boldsymbol{U}(\boldsymbol{x}, t))=\left[S C F_{i}^{\mathrm{N}}(\boldsymbol{x}) \quad S C F_{i}^{\mathrm{MIP}}(\boldsymbol{x}) \quad S C F_{i}^{\mathrm{MOP}}(\boldsymbol{x})\right] \boldsymbol{E} \boldsymbol{B}_{\boldsymbol{x} \boldsymbol{x}}(\boldsymbol{x}) \boldsymbol{u}_{e}(\boldsymbol{x}, t), \quad \forall i
$$

Here $S C F_{i}^{\mathrm{N}}, S C F_{i}^{\mathrm{MIP}}$, and $S C F_{i}^{\mathrm{MOP}}$ are the SCFs for the stresses caused by axial forces, by in-plane moments, and by out-of-plane moments, respectively, for location $i$. The SCFs are explicit functions of the design variables, the connection type, and the loading type. The equations were derived using 3D shell finite element analyses [27, 28]. The fatigue is evaluated between each beam element, but SCFs are only applied to tubular joints.

Restrictions must be enforced on the design variables to ensure reliable results using the HSS method. If a subscript $b$ refers to a brace element, and a subscript $c$ refers to a chord element, the diameters $d$ and thicknesses $t$ must for all relations 


\begin{tabular}{ccccccc}
\hline DLC & $v[\mathrm{~m} / \mathrm{s}]$ & $T I[\%]$ & $H_{s}[\mathrm{~m}]$ & $T_{p}[\mathrm{~s}]$ & $\gamma$ & Occurences/year $[\mathrm{hrs}]$ \\
\hline 1.2 & 6 & 17.5 & 1.18 & 5.76 & 1.0 & 4730.1 \\
& 14 & 14.2 & 1.91 & 6.07 & 1.0 & 3388.1 \\
& 22 & 13.3 & 3.09 & 7.40 & 1.0 & 647.8 \\
\hline
\end{tabular}

Table I. Environmental loading conditions from [30]. $v$ is the mean wind speed, $T I$ is the turbulence intensity, $H_{s}$ is the significant wave height, $T_{p}$ is the wave period, and $\gamma$ is the peak enhancement factor.

satisfy [23]:

$$
\begin{aligned}
\frac{d_{c}}{5} & \leq d_{b} \leq d_{c} \\
\frac{t_{c}}{5} & \leq t_{b} \leq t_{c}
\end{aligned}
$$

Additionally, for each element:

$$
16 t_{e} \leq d_{e} \leq 64 t_{e}, \quad \forall e
$$

These linear constraints are reformulated and collectively referred to as $\boldsymbol{A x} \leq \boldsymbol{b}$.

\subsection{Loading conditions}

One Design Load Case (DLC) from IEC 61400-3 [29] is used, specifically the FLS power production DLC 1.2. Three ten minutes load series are included, see Table I. The three load series are chosen such that there is a low, a medium, and a high mean wind speed series.

A significant amount of fatigue damage occurs around rated wind speed. The NREL 5 MW turbine has a rated wind speed of $11.4 \mathrm{~m} / \mathrm{s}$. However, we experienced numerical instabilities in the applied load deconvolution method around the rated mean wind speed. Consequently, the medium wind speed load case is at $14 \mathrm{~m} / \mathrm{s}$. The load deconvolution method is described in section 2.2.1. Note that buoyancy forces are neglected in all analyses and only one wind direction is applied.

The three load series are linearly scaled to represent the lifetime using meteorological statistics of the K13 deep water site [30]. Accurate load reduction is not a focus area of this work and we instead refer to published literature on the subject, e.g. [31]. The loading conditions are assumed fixed in the optimization.

\subsubsection{Aerodynamic loading}

The application of aerodynamic loads differs among the three analyses. The dynamic analysis in FEDEM Windpower allows for an aero-elastic simulation under turbulent inflow, while the quasi-static and dynamic analyses in JADOP are performed with pre-computed force and moment time-series applied on tower top. The static analysis requires a DEL as described in Section 2.3. FEDEM Windpower is utilized to generate rotor loads (i.e. time-series for forces and moments that are applied in JADOP) that represent the time-dependent rotor loads from an integrated transient analysis. The precomputed rotor loads lead to the same structural response as the integrated analysis that was used to generate these force and moment time-series. An algorithm that is based on a discrete convolution method is used for this purpose.

The method is described for a single degree-of-freedom, but it is straightforward to apply to a multi degree-of-freedom system [32]. The effect of a general load time-history $f(t)$ on a linear system at an arbitrary point in time $t=\tau$ can be considered as the effect to an infinitesimal impulse load. The impulse load causes a response of the system for $t>\tau$ that is given by

$$
d u(t ; \tau)=h(t-\tau) f(\tau) d \tau
$$

$h(t)$ is the Impulse Response Function (IRF). The total response of the system $u(t)$ at time $t$ can therefore be obtained by summing (integrating) all contributions to the response from the loads between times $\tau=0$ to $\tau=t$. This leads to an

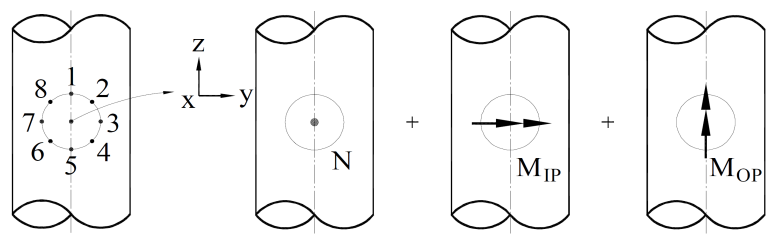

Figure 2. Stresses in the circumference of a weld are determined by superposition of stresses caused by the normal force $\mathrm{N}$, by the in-plane bending moment $\mathrm{M}_{\mathrm{IP}}$, and by the out-of-plane bending moment $\mathrm{M}_{\mathrm{OP}}$. Figure adapted from [23]. 


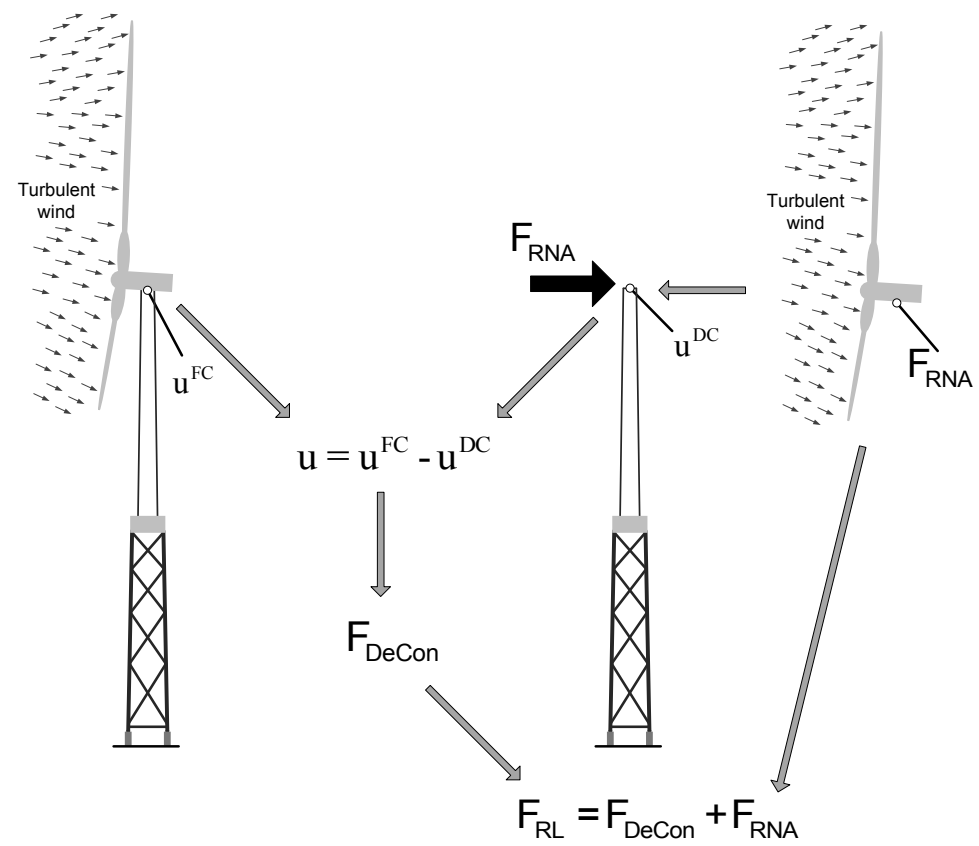

Figure 3. Deconvolution of rotor loads. The differences in tower top displacements from a dynamic analysis with the support structure fully-coupled (FC) to the rotor and from a decoupled (DC) model with precomputed loads $\left(F_{\mathrm{BNA}}\right)$ are used for the deconvolution. The sum of the deconvoluted loads $\left(\mathrm{F}_{\mathrm{DeCon}}\right)$ and the precomputed loads $\left(\mathrm{F}_{\mathrm{RNA}}\right)$ are applied as rotor loads $\left(\mathrm{F}_{\mathrm{RL}}\right)$ in JADOP.

integral expression for the response $u(t)$ that is often referred to as the Duhamel integral [33]:

$$
u(t)=\int_{0}^{t} h(t-\tau) f(\tau) d \tau
$$

The integral can also be written in a discretized version:

$$
u_{n}=\sum_{i=0}^{n-1}\left(h_{n-i} f_{i} \Delta t\right)
$$

Here $u_{n}$ is the discretized displacement at time step $n, h$ the discretized IRF, $f$ the discretized excitation, and $\Delta t$ the time step used in the dynamic simulation. Using the convolution integral in an inverse manner, a so-called deconvolution, allows for computing the input $f(t)$ (e.g. a load time-history applied on a certain degree of freedom) for a given response $u(t)$ (e.g. displacements at the same or a second degree-of-freedom). The transformed equation (9) is given by:

$$
f_{n}=\frac{1}{h_{1}}\left(\frac{u_{n}}{\Delta t}-\sum_{i=1}^{n-1}\left(h_{n+1-i} f_{i}\right)\right)
$$

Equation (10) is prone to numerical issues, especially the minus sign and the fractions can lead to instability of the deconvolution algorithm. A possibility to overcome these numerical issues is used for this study.

A simulation of a standalone model of the RNA with fixed boundary conditions is performed using the same turbulent wind input as the dynamic analysis in FEDEM Windpower. Reaction forces and moments $\left(\mathrm{F}_{\mathrm{RNA}}\right)$ are extracted from the bottom of the RNA and applied at tower top of the same numerical model that is used for the dynamic analysis. However, this second dynamic analysis is performed without aerodynamic calculations, but with the previously calculated forces and moments applied at tower top. The differences in tower top displacements between the dynamic analysis with aeroelastic simulation (fully-coupled) and the decoupled dynamic analysis using the precomputed rotor loads from a standalone turbine simulation $\left(\mathrm{u}^{\mathrm{FC}}-\mathrm{u}^{\mathrm{DC}}\right)$ is afterwards used in equation (10) to calculate a corrective force ( $\left.\mathrm{F}_{\text {DeCon }}\right)$ that is added to the precomputed rotor loads from the standalone turbine simulation. A flow chart of this method is shown in Figure 3.

\subsubsection{Hydrodynamic loading}

In the quasi-static and dynamic approach hydrodynamic loading is included. Based on the wave kinematics for the JONSWAP spectrum [34], the wave forces $f_{w}$ are determined using the Morison equation [35, 36]. Following offshore 
recommendations [29], Wheeler stretching is applied to extend the waves to above mean sea level. The wave forces are applied aligned with the main wind direction. For a fixed body the Morison equation is:

$$
f_{w}(\boldsymbol{x})=\rho_{w} C_{m} V(\boldsymbol{x}) \dot{u}+\frac{1}{2} \rho_{w} C_{d} A(\boldsymbol{x}) u|u|
$$

$\rho_{w}$ is the water density, $C_{m}$ and $C_{d}$ are coefficients for added mass and drag. In this work, the coefficients are assumed fixed and set conservatively to $C_{m}=C_{d}=2 . V$ is the volume of the body and $A$ is the reference area per unit cylinder length. Thus, for a circular cylinder $A=D$ and $V=\frac{1}{4} \pi D^{2}$.

The Morison equation assumes that the water particle velocity $u$ and acceleration $\dot{u}$ are perpendicular to the center axis of the cylinder. However, the jacket members are oriented in a variety of ways, and thus the tangential contributions of the distributed wave forces are neglected.

\subsection{Fatigue analysis}

To simplify the notation, the dependencies on the design variables $\boldsymbol{x}$ and on the state variables $\boldsymbol{U}(\boldsymbol{x}, t)$ are no longer shown. In the quasi-static and dynamic approach, the fatigue analysis follows the recommended practice [23]. The accumulated damage $D_{i}$ in location $i$ is determined using Palmgren-Miner's rule [37, 38] and a bilinear S-N relationship:

$$
\begin{aligned}
D_{i} & =\sum_{j=1}^{3} c_{j} \sum_{k=1}^{n_{k}} \frac{n_{i j k}}{N_{i j k}} \leq \eta \\
\log \left(N_{i j k}\right) & =\log (\bar{a})-m \log \left(\Delta \sigma_{i j k}\right)
\end{aligned}
$$

Here $c_{j}$ is a scaling factor for each load case $j=1,2,3$ to make the data represent 20 years, and is based on meteorological data in [30]. Traditional rainflow-counting is performed to determine the stress range $\Delta \sigma_{i j k}$ [39]. $N_{i j k}$ is the expected cycles to failure at location $i$, load case $j$, and stress state $k$. Likewise, $n_{i j k}$ is the number of load cycles. $n_{k}$ is the number of stress cycles and $\eta=1$ is the allowable damage, also referred to as the utilization factor. (12) constitutes the fatigue constraint equations in the quasi-static and dynamic approaches. Assuming that all steel members are submerged in seawater, the material parameters for tubular joints with cathodic protection are [23]:

$$
\log (\bar{a})=\left\{\begin{array}{ll}
11.764, & \text { if } \Delta \sigma_{i j k} \geq 83.41 \mathrm{MPa} . \\
15.606, & \text { otherwise. }
\end{array} \quad m= \begin{cases}3.0, & \text { if } \Delta \sigma_{i j k} \geq 83.41 \mathrm{MPa} \\
5.0, & \text { otherwise }\end{cases}\right.
$$

In the DEL approach, the structure is subject to a harmonic load with a frequency of $1 \mathrm{~Hz}$ and a load range of $\Delta P^{1 \mathrm{~Hz}}$. The DEL accumulated damage $D_{i}^{1 \mathrm{~Hz}}$ is:

$$
D_{i}^{1 \mathrm{~Hz}}=\frac{n_{T}}{N_{i}^{1 \mathrm{~Hz}}}
$$

$n_{T}=\sum_{j=1}^{3} c_{j} n_{S}$ is the total lifetime, with $n_{S}=600 \mathrm{~s}$ being the simulated time per load case. $N_{i}^{1 \mathrm{~Hz}}$ is the expected number of cycles to failure for the DEL approach. Assuming one-degree-of-freedom loading and quasi-static structural response, the stresses scale linearly with the load, $\sigma_{i}=\alpha_{i} \boldsymbol{P}$. We set $D_{i}^{1 \mathrm{~Hz}}=D_{i}$ and solve for the load range:

$$
\begin{aligned}
\frac{n_{T}\left(\alpha_{i} \Delta \boldsymbol{P}^{1 \mathrm{~Hz}}\right)^{m}}{\bar{a}} & =\sum_{j=1}^{3} c_{j} \sum_{k=1}^{n_{k}} \frac{n_{j k}\left(\alpha_{i} \Delta \boldsymbol{P}_{j k}\right)^{m}}{\bar{a}} \\
\Rightarrow \Delta \boldsymbol{P}^{1 \mathrm{~Hz}} & =\left(\frac{1}{n_{T}} \sum_{j=1}^{3} c_{j} \sum_{k=1}^{n_{k}} n_{j k}\left(\Delta \boldsymbol{P}_{j k}\right)^{m}\right)^{\frac{1}{m}}
\end{aligned}
$$

Note that the DEL approach does not allow for bilinear S-N relations. In this work $\log (\bar{a})=11.764$ and $m=3.0$ are conservatively chosen. The stress range $\Delta \sigma_{i}^{1 \mathrm{~Hz}}$ from the harmonic load is the absolute value of the stress when $\Delta \boldsymbol{P}^{1 \mathrm{~Hz}}$ is applied as a static load. Thus, the DEL accumulated damage is:

$$
D_{i}^{1 \mathrm{~Hz}}=\frac{n_{T}\left(\left|\sigma_{i}^{1 \mathrm{~Hz}}\right|\right)^{m}}{\bar{a}} \leq \eta
$$

This can be reformulated into a stress constraint bounded by a lower $\underline{\Delta \sigma}$ and an upper $\overline{\Delta \sigma}$ stress range limit:

$$
\underline{\Delta \sigma}=-\left(\frac{\eta \bar{a}}{n_{T}}\right)^{\frac{1}{m}} \leq \sigma_{i}^{1 \mathrm{~Hz}} \leq\left(\frac{\eta \bar{a}}{n_{T}}\right)^{\frac{1}{m}}=\overline{\Delta \sigma}
$$


This equation constitutes the DEL constraints.

Although the DEL is generally limited to one-degree-of-freedom loading, two load scenarios that include more loadcomponents are applied based on recommendations in [40]. A thrust dominated load, which in theory should be design driving for the legs, and a torsion dominated load, which is expected to be design driving for the braces.

- Thrust-based: $F_{x}, M_{y}$, and $\frac{1}{2} M_{z}$

- Torsion-based: $\frac{1}{2} F_{x}, \frac{1}{2} M_{y}$ and $M_{z}$

Both load scenarios include all three load cases but neglect wave forces. As two load scenarios are used, there are twice the amount of fatigue constraints, $2 \cdot n_{i}$, in the DEL approach as compared with the other approaches.

\subsection{Frequency analysis}

The eigenfrequencies $\omega_{l}$ are determined by the finite element formulation of a real, symmetric eigenvalue problem:

$$
\boldsymbol{K} \phi_{l}=\lambda_{l} \boldsymbol{M} \phi_{l}, \quad \omega_{l}=\frac{\sqrt{\lambda_{i}}}{2 \pi}, \quad l=1,2, \ldots, n_{l}
$$

Here $\phi_{l}$ and $\lambda_{l}$ represent the eigenvector and eigenvalue, respectively. The eigenvalues have been sorted such that $\lambda_{1}<\lambda_{2}<\ldots<\lambda_{n_{l}}$. Distinct eigenvalues are assumed.

The initial jacket is designed to have the lowest eigenfrequencies in the soft-stiff region, i.e. between the rotor (1P) and blade passing (3P) frequency bands. In the optimization, the first fore-aft and side-to-side frequencies are constrained to lie within these limits with a ten percent safety-margin, i.e. $0.22 \mathrm{~Hz} \leq f_{l} \leq 0.31 \mathrm{~Hz}, l=1,2$. Additionally, the third eigenfrequency is constrained to be above the $3 \mathrm{P}$ frequency. Thus, there are $n_{l}=3$ frequency constraints.

\subsection{Optimization problems}

The minimization of mass problem for the DEL approach is:

$$
\begin{array}{lll}
\underset{\boldsymbol{x} \in \mathbb{R}^{n_{v}}}{\operatorname{minimize}} & f=\rho \sum_{e=1}^{n_{e}} A_{e} l_{e} \\
\text { subject to } & \boldsymbol{A} \boldsymbol{x} \leq \boldsymbol{b} & \\
& \underline{\Delta \sigma} \leq \Delta \sigma_{i}^{1 H z} \leq \overline{\Delta \sigma}, & i=1,2, \ldots, 2 \cdot n_{i} \\
& \underline{\omega_{l}} \leq \omega_{l} \leq \overline{\omega_{l}} & l=1,2, \ldots, n_{l} \\
& \underline{x_{v}} \leq x_{v} \leq \overline{x_{v}}, & v=1,2, \ldots, n_{v}
\end{array}
$$

Here $\rho$ is the material density of S355 steel, and $A_{e}$ and $l_{e}$ are the cross sectional area and length of element $e$, respectively. $n_{e}$ is the total number of elements. $\underline{x_{v}}$ and $\overline{x_{v}}$ are the design variable bounds defined in (27), and $n_{v}$ is the number of design variables. The optimization problem for the quasi-static and dynamic approach is:

$$
\begin{array}{lll}
\underset{\boldsymbol{x} \in \mathbb{R}^{n_{v}}}{\operatorname{minimize}} & f=\rho \sum_{e=1}^{n_{e}} A_{e} l_{e} & \\
\text { subject to } & \boldsymbol{A} \boldsymbol{x} \leq \boldsymbol{b} & \\
& D_{i} \leq \eta, & i=1,2, \ldots, n_{i} \\
& \underline{\omega_{l}} \leq \omega_{l} \leq \overline{\omega_{l}} & l=1,2, \ldots, n_{l} \\
& \underline{x_{v}} \leq x_{v} \leq \overline{x_{v}}, & v=1,2, \ldots, n_{v}
\end{array}
$$

The optimization problems are solved using IPOPT. IPOPT is an open source software package for large-scale nonlinear optimization. It uses a primal-dual interior point method with filters to promote global convergence. Non-default parameters for IPOPT used in the numerical experiments are listed in Table II. The mu strategy is set to adaptive, as this gives faster convergence than the more stable monotonous setting which is default. Instead of using the default nlp scaling method in IPOPT, we chose to scale both objective and constraint functions with $10^{-5}$ based on experiments. The Hessian is approximated using a limited-memory BFGS method, as an analytical Hessian is not easily derived for this problem. Finally, the tolerance is set to $10^{-5}$ based on experience with this type of problem. The maximum number of iterations is set to 100 although this bound is never reached for the problems in this paper. In addition to the convergence criteria in IPOPT, the optimization is set to stop if the relative design change $\Delta \boldsymbol{x}$ in an iteration $(I)$ is below a limit:

$$
\Delta \boldsymbol{x}=\frac{\left\|\boldsymbol{x}^{(I-1)}-\boldsymbol{x}^{(I)}\right\|}{\|\underline{\boldsymbol{x}}-\overline{\boldsymbol{x}}\|} \leq 10^{-5}
$$




\begin{tabular}{lr}
\hline Parameter & Value \\
\hline mu stategy & 'adaptive' \\
nlp scaling method & 'none' \\
hessian approximation & 'limited-memory' \\
tol & $10^{-5}$ \\
max iter & 100 \\
\hline
\end{tabular}

Table II. IPOPT settings different from default.

\subsection{Design sensitivity analysis}

The design sensitivity analysis of the DEL approach is performed using the direct differentiation method [41]. Using the chain rule, the stress sensitivity with respect to a design variable $x_{v}$ is:

$$
\frac{d \sigma_{i}^{1 \mathrm{~Hz}}}{d x_{v}}=\frac{\partial \sigma_{i}^{1 \mathrm{~Hz}}}{\partial x_{v}}+\frac{\partial \sigma_{i}^{1 \mathrm{~Hz}}}{\partial \boldsymbol{U}^{1 \mathrm{~Hz}}} \frac{d \boldsymbol{U}^{1 \mathrm{~Hz}}}{d x_{v}}
$$

The displacement sensitivity is found by differentiating (1) for the static case:

$$
\boldsymbol{K} \frac{d \boldsymbol{U}^{1 \mathrm{~Hz}}}{d x_{v}}=-\frac{d \boldsymbol{K}}{d x_{v}} \boldsymbol{U}^{1 \mathrm{~Hz}}
$$

The loading is assumed design independent, thus $\frac{d P^{1 \mathrm{~Hz}}}{d x_{v}}=0$. The same assumption is made in the quasi-static and dynamic approaches.

Differentiating the quasi-static and dynamic constraint equation (12) with respect to a design variable gives:

$$
\frac{d D_{i}}{d x_{v}}=\frac{\partial D_{i}}{\partial x_{v}}+\sum_{j=1}^{3} \sum_{k=1}^{n_{k}} \frac{\partial D_{i}}{\partial \Delta \boldsymbol{U}_{j k}} \frac{d \Delta \boldsymbol{U}_{j k}}{d x_{v}}
$$

For the quasi-static approach, the full derivative of the displacement range for every load case $j$, and every stress state $k$, are found by:

$$
\boldsymbol{K} \frac{d \Delta \boldsymbol{U}_{j k}}{d x_{v}}=-\frac{d \boldsymbol{K}}{d x_{v}} \Delta \boldsymbol{U}_{j k}, \quad j=1,2,3 \quad k=1, \ldots, n_{k}
$$

It can be seen that the computational cost of the quasi-static approach is much higher than the static approach as this equation needs to be solved many times.

The dynamic design sensitivities differ from the quasi-static approach in computational cost primarily for the displacement sensitivities. The design sensitivity is given by (23), and the displacement sensitivities are:

$$
\boldsymbol{K} \frac{d \Delta \boldsymbol{U}_{j k}}{d x_{v}}=-\frac{d \boldsymbol{M}}{d x_{v}} \Delta \ddot{\boldsymbol{U}}_{j k}-\boldsymbol{M} \frac{d \Delta \ddot{\boldsymbol{U}}_{j k}}{d x_{v}}-\frac{d \boldsymbol{C}}{d x_{v}} \Delta \dot{\boldsymbol{U}}_{j k}-\boldsymbol{C} \frac{d \Delta \dot{\boldsymbol{U}}_{j k}}{d x_{v}}-\frac{d \boldsymbol{K}}{d x_{v}} \Delta \boldsymbol{U}_{j k}, j=1,2,3 k=1, \ldots, n_{k}
$$

In order to solve this differential equation, time integration is necessary. Thus, it is computationally more costly than the quasi-static counterpart. The derivative of the mass and stiffness matrices are computationally cheap. As Rayleigh damping is applied, the derivative of the damping matrix is a weighted sum of the derivative of the mass and stiffness matrices. From the above equations, it is clear that the computational costs of the design sensitivities follow $(22) \ll(24)<(25)$. Note that mode superposition can be applied in the dynamic approach to increase computational efficiency.

Assuming real distinct eigenvalues, the frequency sensitivity is [42]:

$$
\frac{d \lambda_{i}}{d x_{j}}=\phi_{i}^{T}\left(\frac{d \boldsymbol{K}}{d x_{j}}-\lambda_{i} \frac{d \mathbf{M}}{d x_{j}}\right) \phi_{i}
$$

The computational effort involved in the frequency analysis and its sensitivities is negligible.

\section{NUMERICAL EXAMPLES}

This section is comprised of two parts. First, the overall modeling accuracy is investigated, and then optimized designs are evaluated. The numerical examples are based on the OC4 reference jacket [43] with the NREL 5MW turbine [44]. The jacket is discretized using $n_{e}=524$ finite elements and the damage is evaluated in $n_{i}=4192$ locations. The braces and legs are optimized in each section, resulting in $n_{v}=16$ design variables, see Figure 4 and Table III. The design variable bounds are:

$$
300 \mathrm{~mm} \leq d \leq 2000 \mathrm{~mm}, \quad 15 \mathrm{~mm} \leq t \leq 120 \mathrm{~mm}
$$




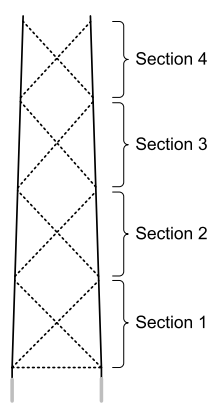

Figure 4. Design variables. Solid line represents legs and dotted line represents braces.

\begin{tabular}{cccc}
\hline Section & Design Variable & $d[\mathrm{~mm}]$ & $t[\mathrm{~mm}]$ \\
\hline 4 & Legs & 1200 & 35 \\
4 & Braces & 800 & 20 \\
3 & Legs & 1200 & 35 \\
3 & Braces & 800 & 20 \\
2 & Legs & 1200 & 35 \\
2 & Braces & 800 & 20 \\
1 & Legs & 1200 & 50 \\
1 & Braces & 800 & 20 \\
\hline
\end{tabular}

Table III. Initial diameters and thicknesses.

\subsection{Comparison of analyses}

To ensure an optimization that presents reliable designs with accumulated damage near the utilization limit, a thorough understanding of the analyses is necessary. In the following, the accuracy of the damage estimates is investigated.

As seen in Table IV, the dynamic approach captures the maximum and mean damage values well. The static approach overpredicts the damage in two locations at section 4 in X-braces perpendicular to the wind-direction on either side of the jacket. Additionally, both the static and quasi-static analyses severely underestimate the damage in most X-braces, especially in X-braces aligned with the wind-direction.

The underestimates are primarily caused by the quasi-static analysis not correctly capturing all out-of-plane displacements in the X-braces. On Figure 5 a spectral analysis of the out-of-plane displacements in an X-brace aligned with the wind-direction in section 3 of the jacket is shown. It is clear that the errors are a direct result of excitations at a structural frequency which cannot be captured by quasi-static modeling. The rotor harmonics are captured well through the applied loading condition (see the 3P, 6P etc. peaks) for all analysis methods. Thus, quasi-static modeling should only be applied if fatigue damage from structural frequencies is negligible or otherwise accounted for. Lastly, note the very good agreement between the dynamic analysis with deconvoluted rotor loads and the integrated aero-elastic simulation in FEDEM Windpower.

Although investigations have shown that the modeling errors are design dependent (as they are primarily due to structural frequencies), a simple correction factor is proposed and tested in an optimization setting for the quasi-static and DEL approaches. The relationship between the mean damage of the applied method as compared with the integrated analysis can be used as a constant constraint scaling factor assumed fixed in all optimization iterations. The scaling values are taken directly from Table IV. For instance, using constraint scaling the fatigue constraint in the quasi-static optimization is given by $\frac{1}{0.49} D_{i} \leq \eta$.

The DEL method is based on the state-of-the-art design process for offshore wind turbine support structures given in [40], which states that "For the conceptual design, wind-only-loads at interface are sufficient". This claim is supported by the findings on Figure 5, as there is little difference in the power spectral densities with or without hydrodynamic loads.

\subsection{Utilization of optimized designs}

The DEL method achieved a reduction in mass of $48.17 \%$ in 42 iterations, see Table V and Figure 6. The highest damage is evaluated using FEDEM Windpower to be 2.09. The poor modeling accuracy in the static analysis in JADOP causes the design to become infeasible by a factor of two at the optimum. The quasi-static optimization reduces the mass by $51.86 \%$ in 53 iterations, with a highest damage of 2.81, see Figure 7. A light design is expected as the quasi-static analysis gives the lowest accumulated damage at the original design. The dynamic approach reduced the mass by $38.20 \%$ in 80 iterations, and has a highest accumulated damage of 0.75 , see Figure 8 . Thus, a feasible design is achieved without load recalculation.

\begin{tabular}{lcc}
\hline Method & Max Damage & Mean Damage \\
\hline Dynamic JADOP & 1.08 & 0.95 \\
Quasi-static JADOP & 0.86 & 0.49 \\
Static JADOP & 1.80 & 0.46 \\
\hline
\end{tabular}

Table IV. Damage caused by aerodynamic loading in the static, in the quasi-static, and in the dynamic approach as compared with FEDEM Windpower. Values are normalized with respect to damage in the integrated analysis. 

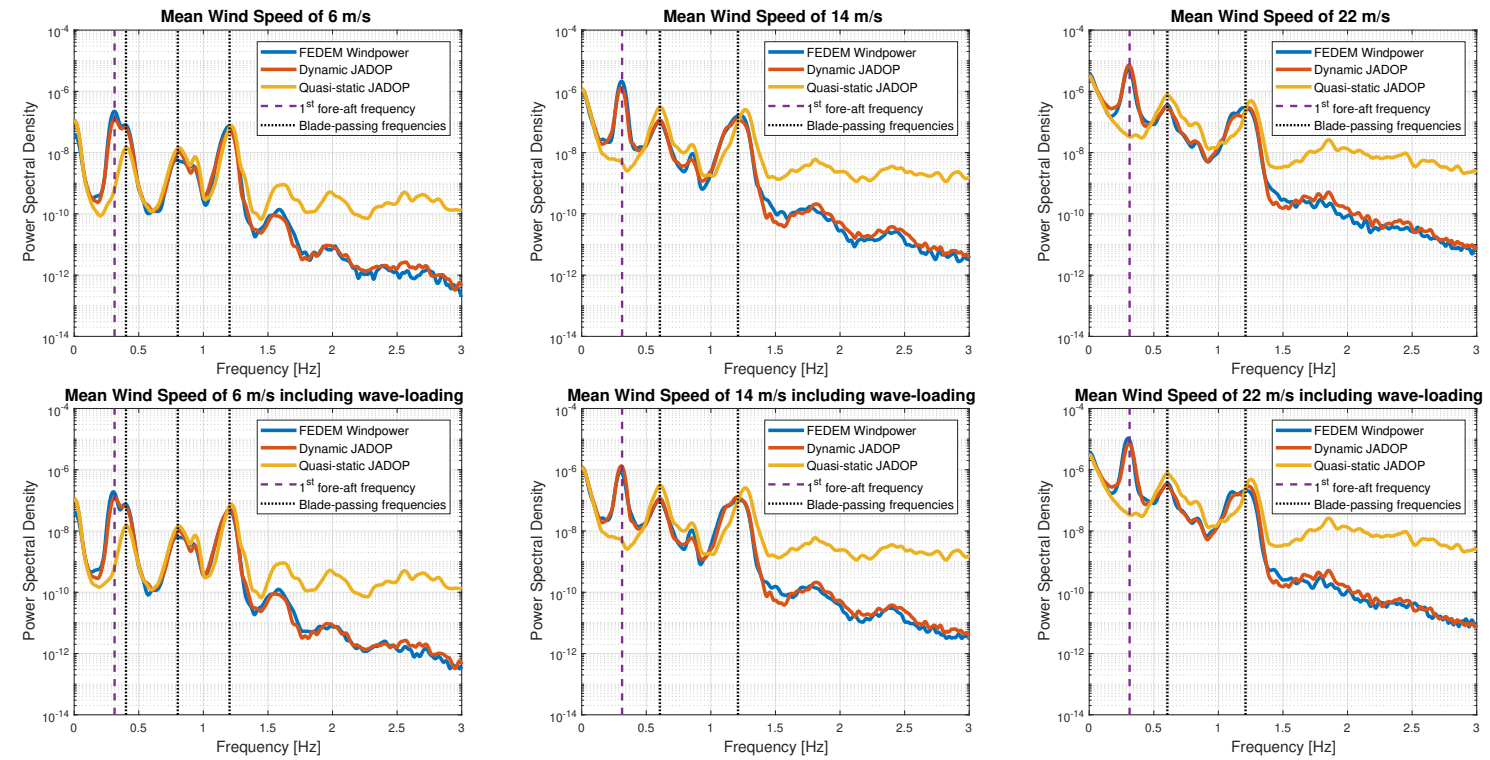

Figure 5. Power spectral densities of out-of-plane displacements in an X-brace in section 3 . Very good agreement is seen between the dynamic analysis in JADOP using deconvoluted loads and the integrated analysis in FEDEM Windpower. Rotor frequencies are captured well by all methods, but structural frequencies cannot be captured by the quasi-static method as highlighted with the first fore-aft frequency.
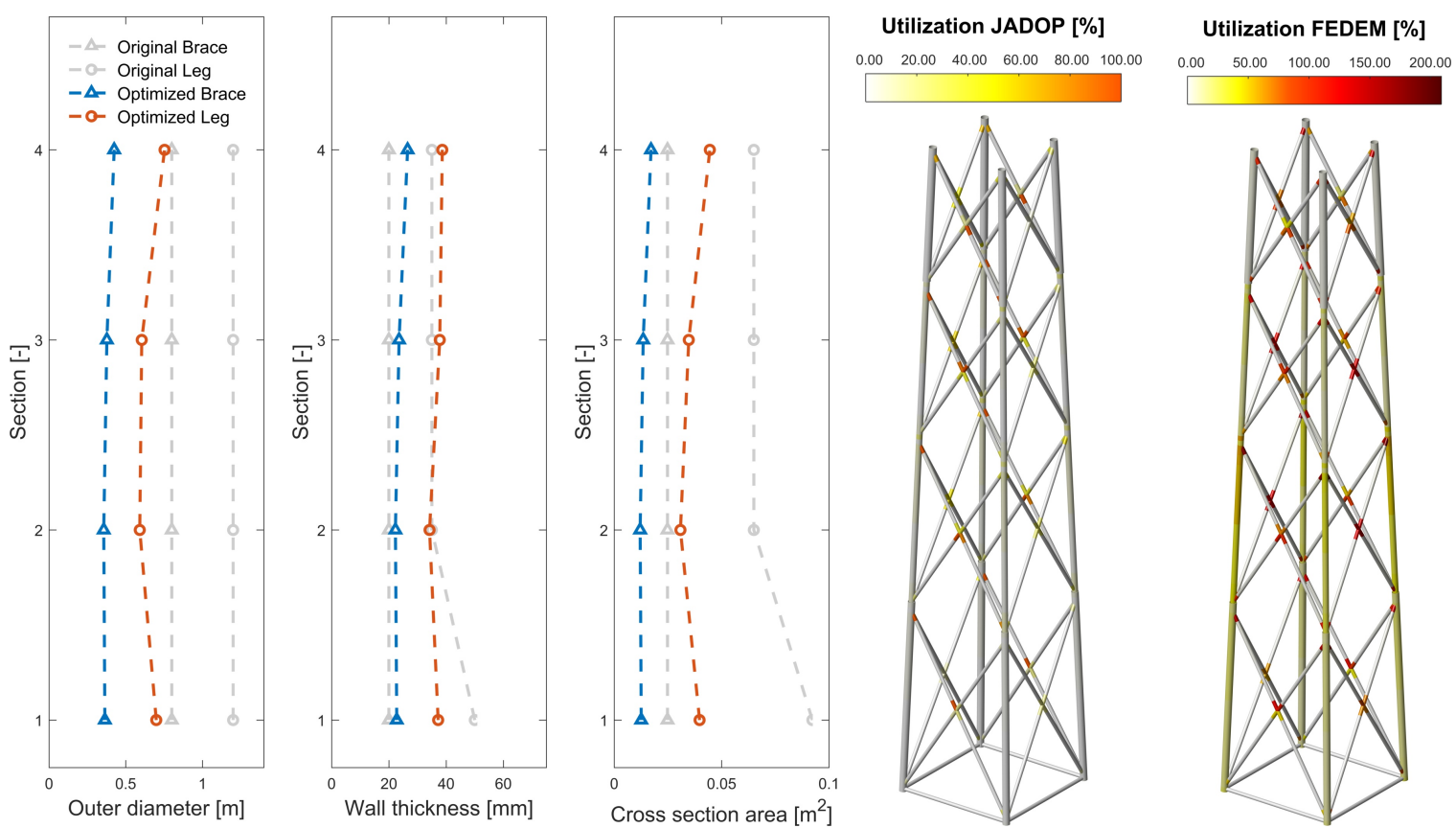

Figure 6. Jacket optimized using the DEL method. The same colormap is used for Figures 6-8.

In Figure 9 the accumulated damage for each approach is illustrated. Due to the linear S-N curve in the DEL approach, the damage levels differ a lot from the quasi-static and dynamic approaches in JADOP. However, when the design is evaluated in FEDEM Windpower, the bilinear S-N curve is applied and the damage levels resemble the other methods more.

Using the constant constraint scaling as defined in Section 3.1, the performance of the DEL and quasi-static optimized designs are improved significantly. In fact, none of the two optimized designs have a highest fatigue damage more than $27 \%$ away from the optimal fatigue value. Considering the highly non-linear behaviour of fatigue, this is satisfactory. 

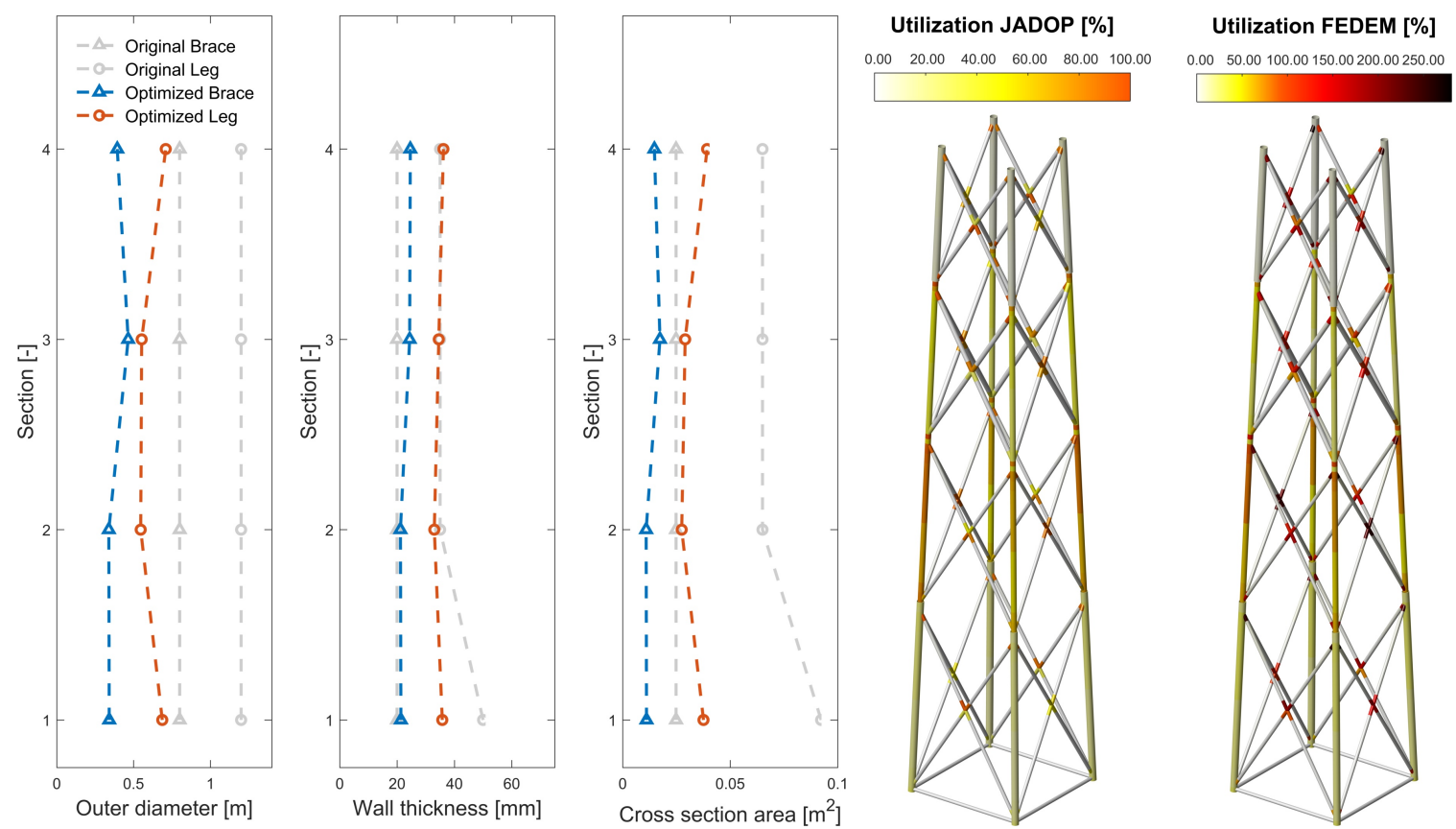

Figure 7. Jacket optimized using the quasi-static method. The same colormap is used for Figures 6-8.
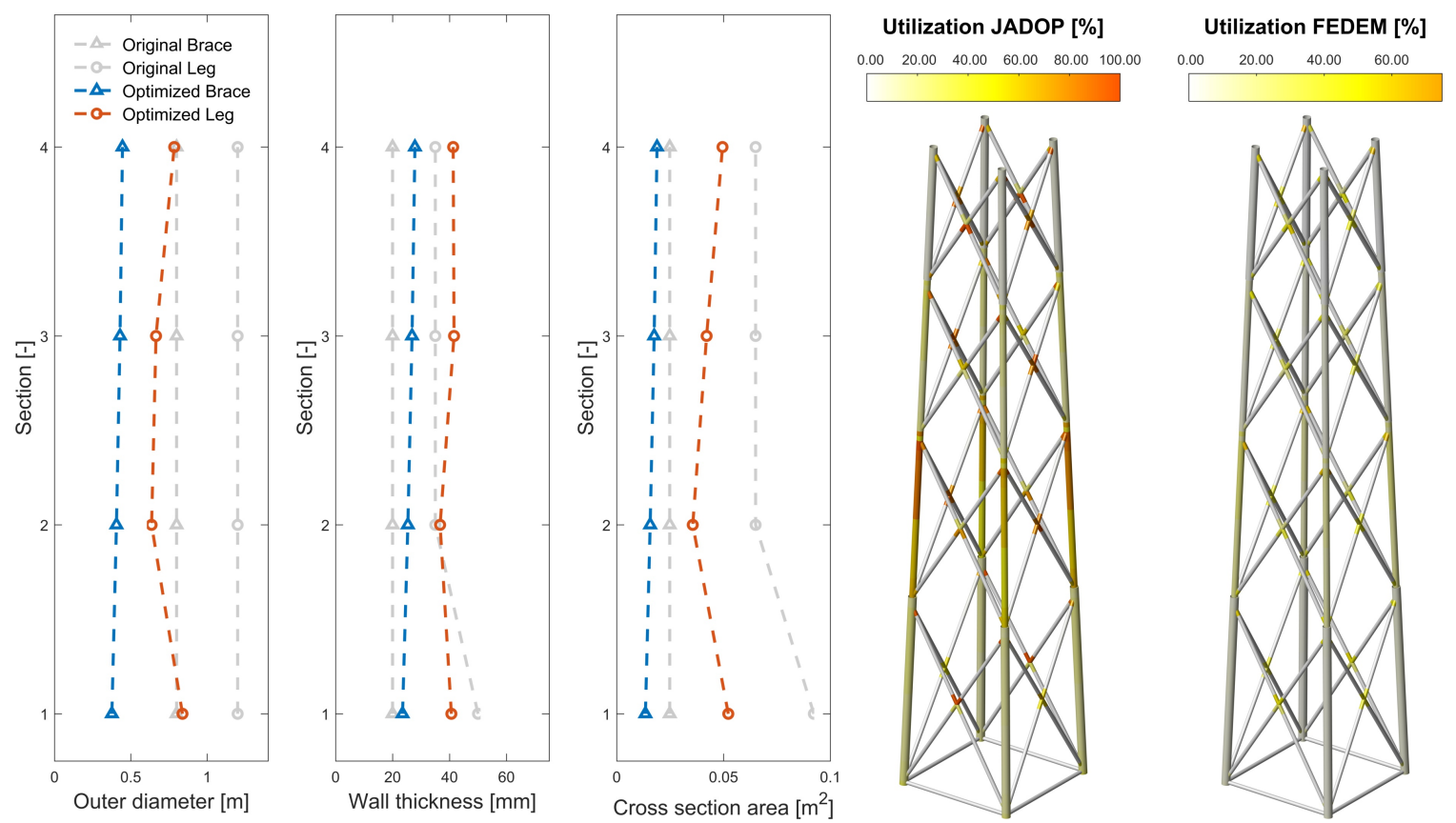

Figure 8. Jacket optimized using the dynamic method. The same colormap is used for Figures 6-8.

All three optimization methods have been tested with a diagonal loading condition, i.e. rotated $45^{\circ}$. It can be seen in Table $\mathrm{V}$ that load direction can have a significant influence on the design.

\subsection{Influence of Stress Concentration Factors}

As seen in Table V, the SCF values are decreased during the optimizations. In general, lowering the SCFs can be achieved by reducing the diameter to thickness ratios of the chords. In Figure 10, SCFs for all joint-types in the initial jacket are plotted with chord thickness $T=0.0675 \mathrm{~m}$ and brace thickness $t=T / 2, t=T / 3$, and $t=T / 4$. 

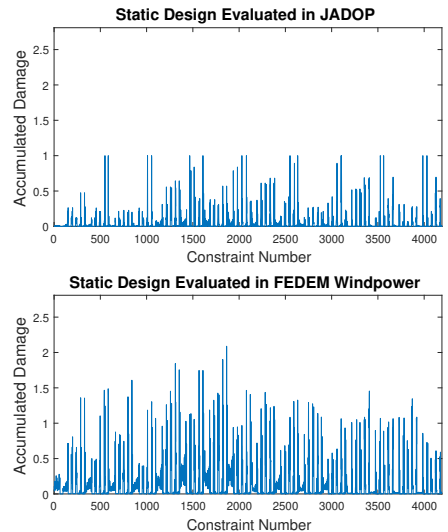
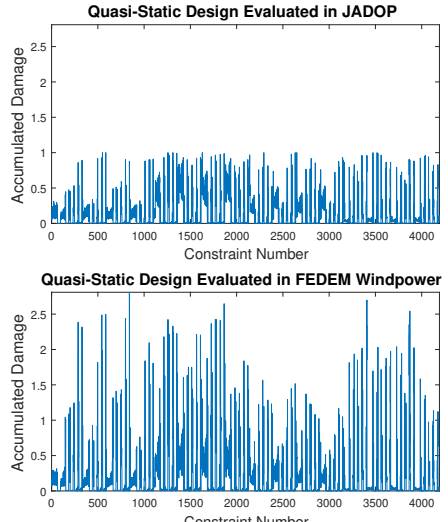
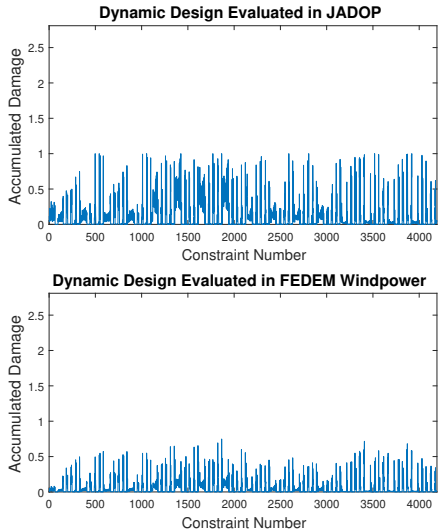

Figure 9. Fatigue damage values. JADOP optimized the quasi-static and dynamic design to a similar damage distribution. However, when evaluated in FEDEM Windpower, it is clear that the quasi-static approach produces an infeasible design.

\begin{tabular}{c|ccccc|ccccccc} 
& \multicolumn{4}{|c|}{ Optimization Settings } & \multicolumn{6}{c}{ Optimization Results } \\
\hline \multirow{2}{*}{ Method } & $\begin{array}{c}\text { Freq. } \\
\text { constr. }\end{array}$ & $\begin{array}{c}\text { Wave- } \\
\text { loads }\end{array}$ & $\begin{array}{c}\text { Load } \\
\text { angle }\left[^{\circ} \text { ] }\right.\end{array}$ & $\begin{array}{c}\text { Constraint } \\
\text { scaling }\end{array}$ & $\begin{array}{c}\text { No of } \\
\text { Iter. }\end{array}$ & $\begin{array}{c}\text { Max } \\
\text { dmg. }\end{array}$ & $\begin{array}{c}\text { Max } \\
\text { SCF }\end{array}$ & $\begin{array}{c}\text { Mean } \\
\text { SCF }\end{array}$ & $\begin{array}{c}\text { Lowest } \\
\text { freq. [Hz] }\end{array}$ & $\begin{array}{c}\text { Mass red. } \\
{[\%]}\end{array}$ & $\begin{array}{c}\text { Mass } \\
\text { [ton] }\end{array}$ \\
\hline Initial Design & & & & & & & 6.54 & 3.21 & 0.31 & 540.21 \\
\hline \multirow{2}{*}{ DEL } & Yes & No & 0 & - & 42 & 2.09 & 3.63 & 2.23 & 0.26 & 48.17 & 279.97 \\
& No & No & 0 & - & 51 & - & - & - & - & 48.17 & 279.97 \\
& Yes & No & 45 & - & 35 & 1.88 & 3.75 & 2.36 & 0.27 & 45.29 & 295.53 \\
& Yes & No & 0 & Yes & 39 & 0.86 & 3.67 & 2.25 & 0.27 & 38.79 & 330.67 \\
\hline \multirow{2}{*}{ Quasi-static } & Yes & Yes & 0 & - & 53 & 2.81 & 3.56 & 2.24 & 0.25 & 51.86 & 260.04 \\
& Yes & Yes & 45 & - & 100 & 2.33 & 3.57 & 2.31 & 0.26 & 48.73 & 276.98 \\
& Yes & No & 0 & - & 42 & 3.09 & 3.48 & 2.19 & 0.25 & 54.02 & 248.39 \\
& No & No & 0 & - & 66 & - & - & - & - & 54.02 & 248.39 \\
& Yes & Yes & 0 & Yes & 55 & 1.27 & 3.51 & 2.26 & 0.26 & 44.04 & 302.28 \\
\hline \multirow{2}{*}{ Dynamic } & Yes & Yes & 0 & - & 80 & 0.75 & 3.57 & 2.25 & 0.27 & 38.20 & 333.88 \\
& Yes & Yes & 45 & - & 36 & 0.70 & 3.45 & 2.33 & 0.28 & 35.00 & 351.11 \\
& Yes & No & 0 & - & 53 & 0.83 & 3.58 & 2.19 & 0.27 & 39.96 & 324.36 \\
& No & No & 0 & - & 51 & - & - & - & - & 39.96 & 324.36 \\
\hline
\end{tabular}

Table V. Different optimization scenarios run. Excluding frequency constraints did not affect the optimized structure. Including waveloading does not add significant weight to the structure. Changing the load direction has a large influence on the overall mass. The dynamic approach is superior, but with constant constraint scaling, each method performs well.

The amount of fatigue evaluations is prescribed in standards to be eight equally spaced locations in the circumference of the weld. However, previous studies show that the highest fatigue damage is not necessarily captured when using eight hotspots [45]. In this work the fatigue damage was captured within an acceptable level of accuracy using eight hotspots. Thus, this design-dependent issue is not further investigated.

\section{DISCUSSION}

The accuracy of the analyses is reflected in the optimizations. The DEL method overestimates the damage significantly in a few hotspots. Thus, the method produces a design closer to feasibility than the quasi-static approach. However, the damage is better distributed in the quasi-static design. The dynamic approach generates a feasible design without a single load recalculation during the optimization.

Although the structures are reduced to almost half the total weight, the damage caused by the updated aero- and hydrodynamic loading is not changed significantly as compared with the damage caused by the initial loading conditions. This is key to an effective gradient-based optimization approach. Partly because recalculating loads in each design iteration is computationally expensive, and partly because it is not possible to determine the design sensitivity of the loading condition analytically. If loads are very sensitive to design changes, and load sensitivities are not provided, this can prevent 

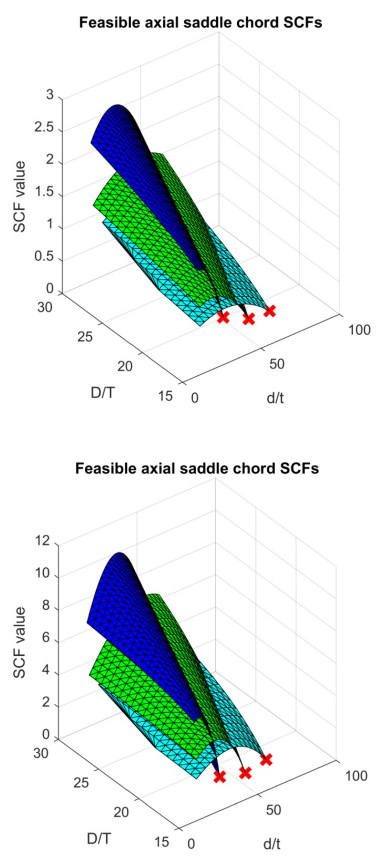

Feasible axial saddle chord SCFs

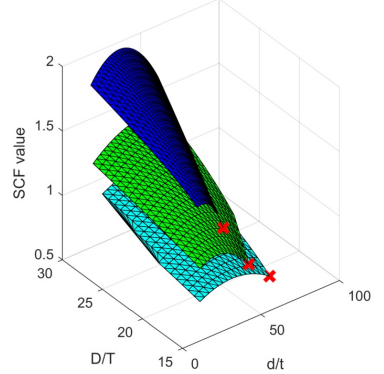

Feasible axial crown chord SCFs

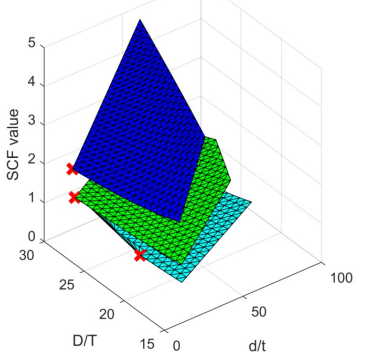

(a)

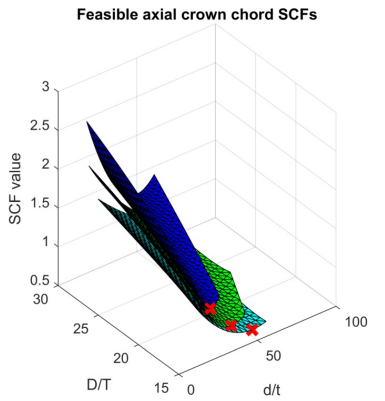

a) Y-joint
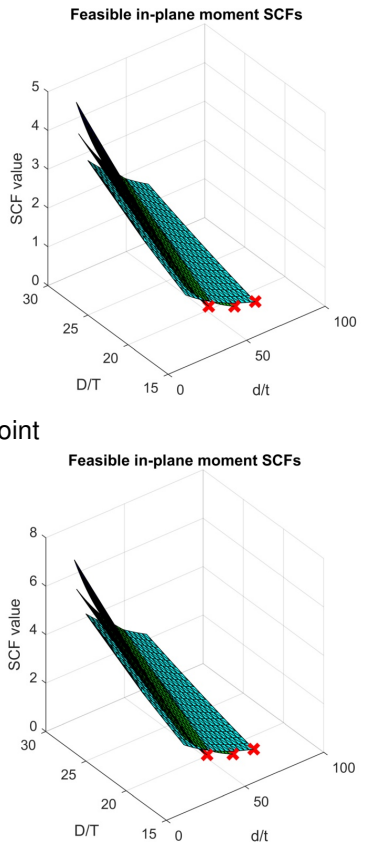

(b)

(b) X-joint
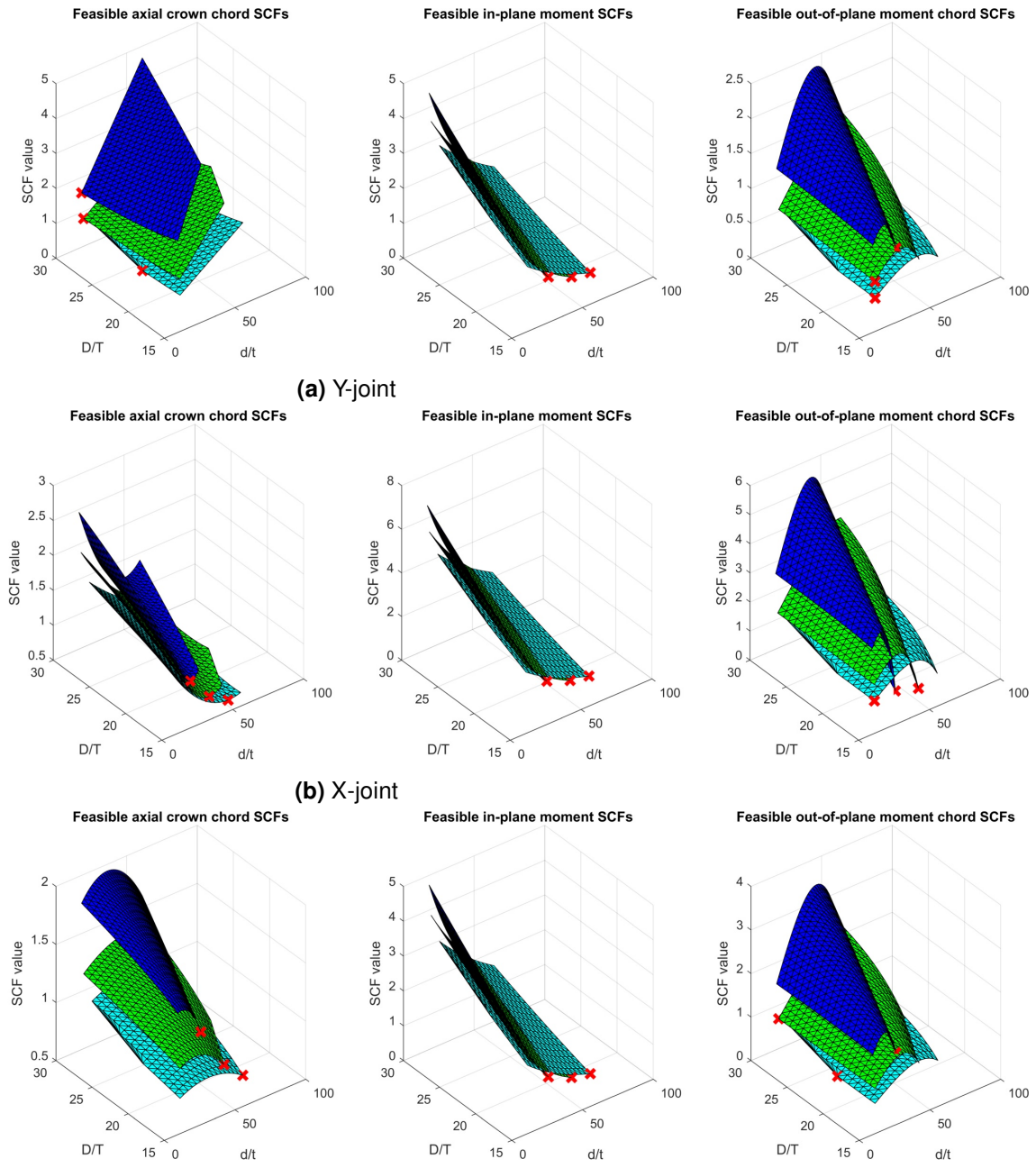

Feasible out-of-plane moment chord SCFs

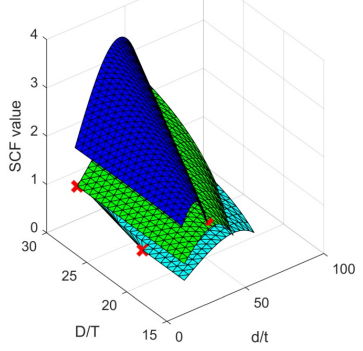

(c) K-joint

Figure 10. SCF values for three different thickness relationships between the chord thickness, $T$, and the brace thickness, $t$. In blue $t$ $=T / 2$, in green $t=T / 3$, and in cyan $t=T / 4$. The red cross represents the lowest SCF value for the given thickness relations.

the optimization from converging. It is possible to use finite differences to estimate the loading sensitivity, but this is very computationally costly.

Restarting the design process with an optimized design as initial guess and applying updated rotor and wave-loads can, ultimately, produce a design that has full fatigue utilization. The computational cost of this process is greatly reduced when the loading condition does not change significantly.

Hydrodynamic loading does not influence the optimized designs much. However, load direction is important for the design as seen in Table V. Therefore, it is recommended to include loads in different directions.

Using constraint scaling, better designs were achieved for both the DEL and quasi-static methods. The constant constraint scaling method is an adjustment of the estimated fatigue damage and does not provide optimal designs. With the constant constraint scaling factor, the static and quasi-static approaches generate more realistic and less non-conservative designs, but the trends not captured by the simpler analyses may not be accounted for in the optimized designs.

In the present work a strict convergence criterion is applied. When bilinear S-N curves are used, oscillations near optimum could be observed in some cases. Smoothing of the bilinear S-N curve can be applied to address this issue.

None of the presented observations may be generic, and it is therefore important to have a deep knowledge of the mechanics of jackets even for conceptual and preliminary design optimizations. Many parameters such as yaw misalignment, wind speed, different jackets and turbines, soil-structure interaction etc. have not been investigated. Additionally, the study is limited to fatigue constraints using a small set of loads, and thus the effects of ULS and manufacturing constraints are not investigated. 
The usefulness of a numerical design optimization of a jacket structure in an industrial work-flow is not addressed in most literature. It is important that the optimization provides a design comparable or even better than a design based on expert knowledge and experience. Most optimizations of support structures focus on a very limited set of structural constraints, while experts have the bigger picture in mind. In other words, much work in the field of gradient-based optimization of jacket structures is still to be done.

\section{CONCLUSION}

Three different state-of-the-art analytical gradient-based optimization approaches to minimizing the mass of a jacket structure with fatigue and frequency constraints are explored within the same framework. The three methods can be defined by their analysis methods, i.e. (i) damage equivalent loading using static analysis, (ii) time-dependent loading using quasi-static analysis, and (iii) time-dependent loading using transient analysis. By using all three methods within the same framework, a fair comparison between the methods is established.

The force and moment time-series applied in the optimizations are determined from a fully integrated analysis using a so-called deconvolution method. The method is evaluated and is shown to be accurate in the examples studied.

The performance and applicability of each method are critically studied in state-of-the-art commercial software. By studying the response and fatigue damage levels of both the initial reference design and the optimized designs, insight into important structural and modeling parameters is provided. Several assumptions and limitations are used to limit the study, e.g. large load reductions, simplified load directions, and simplified boundary conditions. Because of the assumptions, the achieved mass reductions are likely an overestimate of what is actually achievable in a more realistic design optimization. Consequently, the optimized designs must not be considered as realistic jacket structures, but are intended as base of comparison between the optimization approaches. Not surprisingly, the investigation shows that the transient approach is superior, but the investigation also shows how the simpler and faster approaches can be viable with precaution and strong insight into the problem.

\section{ACKNOWLEDGEMENTS}

This research is part of the project ABYSS - Advancing BeYond Shallow waterS - Optimal design of offshore wind turbine support structures, sponsored by the Danish Council for Strategic Research, Grant no. 1305-00020B. This support is gratefully acknowledged. Additionally, support by NOWITECH, the Norwegian Research Centre for Offshore Wind Technology, is gratefully acknowledged (Research council of Norway, Contract no. 193823). We thank in particular Fedem Technology AS for providing software licenses and quick and helpful feedback. Lastly, the authors would like to thank Alexander Verbart for his work on JADOP.

\section{REFERENCES}

1. Sun X, Huang D, Wu G. The current state of offshore wind energy technology development. Energy 2012; 41(1):298312.

2. International Energy Agency. Technology roadmap - Wind energy. Technical Report, Paris 2013.

3. Burton T, Sharpe D, Jenkins N, Bossanyi E. Wind Energy Handbook. 2nd editio edn., John Wiley \& Sons, 2001.

4. The Carbon Trust. Offshore Wind Power: Big Challenge, Big Opportunity: Maximising the Environmental, Economic and Security Benefits. Technical Report 2008.

5. The Crown Estate. Offshore Wind Cost Reduction: Pathways Study. Technical Report 2012.

6. Yoshida S. Wind Turbine Tower Optimization Method Using a Genetic Algorithm. Wind Engineering 2006; 30(6):453-470.

7. Uys PE, Farkas J, Jármai K, van Tonder F. Optimisation of a steel tower for a wind turbine structure. Engineering Structures jul 2007; 29(7):1337-1342.

8. Thiry A, Bair L, Buldgen GR, Rigo P. Optimization of monopile offshore wind structures. Proceedings of the 3rd international conference on marine structures, 2011; 633-642.

9. Gentils T, Wang L, Kolios A. Integrated structural optimisation of offshore wind turbine support structures based on finite element analysis and genetic algorithm. Applied Energy 2017; 199:187-204.

10. Long H, Moe G, Fischer T. Lattice towers for bottom-fixed offshore wind turbines in the ultimate limit state: variation of some geometric parameters. Journal of Offshore Mechanics and Arctic Engineering dec 2011; 134(2):21 202.

11. Long H, Moe G. Preliminary design of bottom-fixed lattice offshore wind turbine towers in the fatigue limit state by the frequency domain method. Journal of Offshore Mechanics and Arctic Engineering feb 2012; 134(3):31 902. 
12. Zwick D, Muskulus M, Moe G. Iterative optimization approach for the design of full-height lattice towers for offshore wind turbines. Energy Procedia 2012; 24(0):297-304.

13. Pasamontes LB, Torres FG, Zwick D, Schafhirt S, Muskulus M. Support structure optimization for offshore wind turbines with a genetic algorithm. Proceedings of the ASME 2014 33rd Internation Conference on Ocean, Offshore and Arctic Engineering, San Fransisco, 2014; 1-7.

14. Schafhirt S, Zwick D, Muskulus M. Reanalysis of Jacket Support Structure for Computer-Aided Optimization of Offshore Wind Turbines with a Genetic Algorithm. Proceedings of the Twenty-fourth International Ocean and Polar Engineering Conference, Busan, 2014; 234-241.

15. Schafhirt S, Zwick D, Muskulus M. Two-stage local optimization of lattice type support structures for offshore wind turbines. Ocean Engineering 2016; 117:163-173.

16. Ashuri T, Zaaijer MB, Martins J, van Bussel GJW, van Kuik GAM. Multidisciplinary design optimization of offshore wind turbines for minimum levelized cost of energy. Renewable Energy 2014; 68:893-905.

17. Ashuri T, Ponnurangam C, Zhang J, Rotea M. Integrated Layout and Support Structure Optimization for Offshore Wind Farm Design. Journal of Physics: Conference Series, vol. 753, 2016; 92011.

18. Chew Kh, Muskulus M, Narasimalu S, Tai K, Ng EYK. Fatigue sensitivity analysis of offshore wind turbine structures. Proceedings of WCSMO-11 2015; :798-803.

19. Oest J, Sørensen R, Overgaard LCT, Lund E. Structural optimization with fatigue and ultimate limit constraints of jacket structures for large offshore wind turbines. Structural and Multidisciplinary Optimization 2017; 55(3):779_ 793.

20. Chew KH, Tai K, Ng EYK, Muskulus M. Optimization of offshore wind turbine support structures using an analytical gradient-based method. Energy Procedia 2015; 80:100-107.

21. Chew KH, Tai K, Ng EYK, Muskulus M. Analytical gradient-based optimization of offshore wind turbine substructures under fatigue and extreme loads. Marine Structures 2016; 47:23-41.

22. Muskulus M, Schafhirt S. Design optimization of wind turbine support structures - a review. Journal of Ocean and Wind Energy 2014; 1(1):12-22.

23. DNV. RP-C203: Fatigue design of offshore steel structures. Det Norske Veritas: Høvik, 2014.

24. Freebury G, Musial W. Determining equivalent damage loading for full-scale wind turbine blade fatigue tests. 2000 ASME Wind Energy Symposium, 2000; 50.

25. Wächter A, Biegler LT. On the Implementation of a Primal-Dual Interior Point Filter Line Search Algorithm for Large-Scale Nonlinear Programming. Mathematical Programming 2006; 106(1):25-57.

26. Cook RD, Malkus DS, Plesha ME, Witt RJ. Concepts and applications of finite element analysis. 4th ed. edn., John Wiley \& Sons: New York, N.Y., 2002.

27. Efthymiou M, Durkin S. Stress concentrations in T/Y and gap/overlap K-joints. Proceedings of the 4th International Conference on Behaviour of Offshore Structures 1985; :1-12.

28. Efthymiou M. Development of SCF formulae and generalised influence functions for use in fatigue analysis. Proceedings of the Offshore Tubular Joints Conference 1988; :1-13.

29. IEC. IEC 61400-3 Wind Turbines - Part 3: Design requirements for offshore wind turbines. International Electrotechnical Commission: Geneva, 2009.

30. Fischer T, de Vries W, Schmidt B. UpWind Design Basis. Technical Report 2010.

31. Zwick D, Muskulus M. Simplified fatigue load assessment in offshore wind turbine structural analysis. Wind Energy 2015; 19(2):265-278.

32. Passon P, Branner K. Load calculation methods for offshore wind turbine foundations. Ships and Offshore Structures 2013; 9(4):433-449.

33. Clough RW, Penzien J. Dynamics of Structures. McGraw-Hill, 1975.

34. Hasselmann K, Barnett TP, Bouws E, Carlson H, Cartwright DE, Enke K, Ewing JA, Gienapp H, Hasselmann DE, Kruseman P, et al. . Measurements of Wind-Wave Growth and Swell Decay during the Joint North Sea Wave Project (JONSWAP). Technical Report 801973.

35. Morison JR, O'Brien MP, Johnson J, Schaaf S. The Force Exerted by Surface Waves on Piles. Journal of Petroleum Technology 1950; 2(5):149-154.

36. Schløer S, Castillo LG, Fejerskov M, Stroescu E, Bredmose H. A model for Quick Load Analysis for monopile-type offshore wind turbine substructures. Journal of Physics: Conference Series 2016; 753(9):92 008.

37. Palmgren AG. Die Lebensdauer von Kugellagern. Zeitschrift des Vereines Deutscher Ingenieure 1924; 14:339-341.

38. Miner MA. Cumulative Damage in Fatigue. Journal of Applied Mechanics 1945; 12(3):A159-A164.

39. Matsuishi M, Endo T. Fatigue of metals subjected to varying stress. Japan Society of Mechanical Engineering 1968; 68(2):37-40.

40. Seidel M, Voormeeren S, van der Steen JB. State-of-the-art design processes for offshore wind turbine support structures. Stahlbau 2016; 85(9):583-590. 
41. Tortorelli D, Michaleris P. Design sensitivity analysis: Overview and review. Inverse Problems in Science and Engineering 1994; 1(1):71-105.

42. Seyranian AP, Lund E, Olhoff N. Multiple eigenvalues in structural optimization problems. Structural optimization 1994; 8(4):207-227.

43. Vorpahl F, Kaufer D, Popko W. Description of a basic model of the "Upwind reference jacket" for code camparison in the OC4 project under IEA Wind Annex 30". Technical Report, Institute for Wind Energy and Energy Systems Technology 2011.

44. Jonkman J, Butterfield S, Musial W, Scott G. Definition of a 5-MW reference wind turbine for offshore system development. Technical Report February 2009.

45. Hammerstad BH, Schafhirt S, Muskulus M. On Fatigue Damage Assessment for Offshore Support Structures with Tubular Joints. Energy Procedia 2016; 94:339-346. 